\title{
Effect of Different Micronutrients on Turmeric Variety Suranjana in Terai Region of West Bengal, India
}

\author{
S. Datta*, S. Chakraborty, J.C. Jana, A. Debnath, M.K. Roy and S. Haque \\ Uttar Banga Krishi Viswavidyalaya, Pundibari, Coochbehar, 736165, India \\ *Corresponding author
}

\begin{abstract}
A B S T R A C T
An experiment was undertaken at Uttar Banga Krishi Viswavidyalaya, Pundibari, West Bengal to study the effect of boron, iron, manganese and zinc on the growth and yield of Keywords

Micronutrients,

Boron, Iron,

Manganese,

Zinc,

Turmeric, Yield .

Article Info

Accepted:

17 April 2017

Available Online:

10 May 2017 turmeric during the year of 2010-11, 2011-12, 2012-13 and 2015-16. The experiment was laid out in randomized block design with three replications. Different treatments of the experiment were $\mathrm{T}_{1}$ - control (no micronutrients), $\mathrm{T}_{2}$ - soil application of boron (as borax) @ $25 \mathrm{Kg} \mathrm{ha}^{-1}, \mathrm{~T}_{3}$ - soil application of manganese (as $\left.\mathrm{MnSO}_{4}\right) @ 25 \mathrm{Kg} \mathrm{ha}^{-1}, \mathrm{~T}_{4}$ - soil application of iron (as $\mathrm{Fe}_{2} \mathrm{SO}_{4}$ ) @ $25 \mathrm{Kg} \mathrm{ha}^{-1}, \mathrm{~T}_{5}$ - soil application of zinc (as $\mathrm{ZnSO}_{4}$ ) @ $25 \mathrm{Kg} \mathrm{ha}^{-1}, \mathrm{~T}_{6}$ - foliar spray of manganese $\left(\right.$ as $\left.\mathrm{MnSO}_{4}\right) @ 0.5 \%$ after 60 and 90 days of planting, $\mathrm{T}_{7}$ - foliar spray of iron $\left(\mathrm{as}_{2} \mathrm{Fe}_{4}\right) @ 0.5 \%$ after 60 and 90 days of planting, $\mathrm{T}_{8}$ foliar spray of boron (as borax) @ 0.5\% after 60 and 90 days of planting and $\mathrm{T}_{9}$ - foliar spray of zinc (as $\left.\mathrm{ZnSO}_{4}\right) @ 0.5 \%$ after 60 and 90 days of planting. The results revealed that soil application of boron (as borax) @ 25 $\left.\mathrm{Kg} \mathrm{ha}^{-1}\right)$ gave the highest yield $(11.13 \mathrm{~kg} / 3$ $\mathrm{m}^{2}$ and $22.45 \mathrm{t} / \mathrm{ha}$ ) which was also statistically at par with foliar spray of boron as borax @ $0.5 \%$ at 60 and 90 days after planting $\left(10.59 \mathrm{~kg} / 3 \mathrm{~m}^{2}\right.$ and $\left.21.36 \mathrm{t} / \mathrm{ha}\right)$. The lowest yield $\left(7.17 \mathrm{~kg} / 3 \mathrm{~m}^{2}\right.$ and $14.45 \mathrm{t} / \mathrm{ha}$ ).was recorded in the control treatment (i.e. without micronutrient application). The highest cost benefit ratio (1: 2.04) was recorded with soil application of boron (as borax) @ $25 \mathrm{Kg} \mathrm{ha}^{-1}$ followed by foliar spray of boron (1:1.95) (as borax) @ $0.5 \%$ at 60 and 90 days after planting.
\end{abstract}

\section{Introduction}

Turmeric (Curcuma longa L.) is an important rhizomatous spice crop of India and World too. India is a leading producer, consumer and exporter of turmeric in the world. India is the major producer and exporter of turmeric and earned a foreign exchange of 2000 million \$ (Anonymous, 2012). Apart from its spice and medicinal value it is also used in the dye, food and cosmetic industries. It is also used in the auspicious religious occasions. Turmeric inhibits the development of cataracts, breast cancer, colon cancer, and lymphoma (Devi et $a l ., 2011)$. In India it is cultivated with an area of 1.95 lakh hectare (ha) with a production of 9.99 lakh tonnes. In West Bengal, it is cultivated with an area of 15.8 thousand ha and production of 42 thousand tonnes. Productivity of turmeric in West Bengal is quite low (2.66 tones/ha) compared to national average (5.11 tones/ha). It is a soil exhausting crop and application of N, P and K are recommended for its cultivation. Normally micronutrients do not find a place in the nutrient recommendations. However, high rhizome yields in turmeric with increasing concentrations of foliar sprays of 
magnesium sulphate in the acidic soils of Meghalaya have been reported (Chandra et $a l$. , 1997). Turmeric is highly responsive to chemical fertilizers. In addition, micronutrients fertilizers are mostly liable to reduce disease incidence and enhance durability of the post harvest life of ginger and turmeric (Halder et al., 2007). (Banafer et al., 1995), (Gupta and Singer, 1998), (Roy et al., 1992) stated in therir reports that ginger and turmeric are highly responsive to chemical fertilzers and increased the growth and rhizome yield with increments of fertilizer rates. Bose et al., (2008) reported that inclusion of $\mathrm{S}$ and $\mathrm{Mg}$ in the fertilization schedule greatly improved the fresh yields of turmeric in the depleted red lateritic soils of West Bengal. Similarly, Kavitha (2012) revealed that in the rhizomatous crop kacholam (Kaempferia galanga L.), top dressing of sulphur and magnesium resulted in higher rhizome yields. Recent research has shown that application of $\mathrm{Fe}$ significantly increased in yield of crops. Chhibba et al., (2007) reported that the foliar application of Fe increased yield and Fe concentration in fenugreek. Beneficial effect of Effect of $\mathrm{Zn}$, $\mathrm{Fe}$ and $\mathrm{B}$ on growth and yield of ginger was reported by Roy et al., (1992). Halder et al (2007) reported the beneficial effect of zinc and boron on turmeric yield and quality. Very information is available with respect to the effect of boron, iron, manganese and zinc on the growth and yield of turmeric, with keeping this views, the present experiment was undertaken with the effect of boron, iron, manganese and zinc on the growth and yield of turmeric.

\section{Materials and Methods}

An investigation was undertaken to study the effect of boron, iron, manganese and zinc on the growth and yield of turmeric in Uttar Banga Krishi Viswavidyalaya, Pundibari, Cooch Behar, West Bengal during the year of
2010-11, 2011-12, 2012-13 and 2015-16. The experimental soil was sandy clay loam having $p^{\mathrm{H}} 5.6,0.90 \%$ organic carbon, $131.45 \mathrm{~kg} / \mathrm{ha}$ available nitrogen, $47.38 \mathrm{~kg} / \mathrm{ha}$ available phosphorus and $65.82 \mathrm{~kg} / \mathrm{ha}$ potash. The climatic condition of this region is subtropical humid in nature. The experiment was laid out in Randomized Block Design with five replications. Raised beds of $3 \mathrm{~m}$ X $1 \mathrm{~m}$ size and $15 \mathrm{~cm}$ height were prepared. Turmeric rhizome of the variety Suranjana was planted with a spacing of $30 \mathrm{~cm} \times 20 \mathrm{~cm}$ during the first week of May 2010, 2011, 2012 and 2015, respectively. The experiment was laid out in randomized block design with 9 treatments and three replications. The treatment details were $\mathrm{T}_{1}$ - control (no micronutrients), $\mathrm{T}_{2}$ - soil application of boron (as borax)@25Kg ha ${ }^{-1}, \mathrm{~T}_{3}$ - soil application of manganese (as $\mathrm{MnSO}_{4}$ ) @ 25Kg ha ${ }^{-1}, \mathrm{~T}_{4}$ soil application of iron (as $\left.\mathrm{Fe}_{2} \mathrm{SO}_{4}\right)$ @ $25 \mathrm{Kg}$ ha $^{-1}, \mathrm{~T}_{5}$ - soil application of zinc (as $\mathrm{ZnSO}_{4}$ ) @ $25 \mathrm{Kg} \mathrm{ha}^{-1}, \mathrm{~T}_{6}$ - foliar spray of manganese (as $\mathrm{MnSO}_{4}$ ) @ $0.5 \%$ after 60 and 90 days of planting, $\mathrm{T}_{7}$ - foliar spray of iron (as $\mathrm{Fe}_{2} \mathrm{SO}_{4}$ ) @ $0.5 \%$ after 60 and 90 days of planting, $\mathrm{T}_{8}$ foliar spray of boron (as borax) @ 0.5\% after 60 and 90 days of planting and $\mathrm{T}_{9}$ - foliar spray of zinc (as $\mathrm{ZnSO}_{4}$ ) @ $0.5 \%$ after 60 and 90 days of planting. Full dose of Farm yard manure @ 15 t/ha was applied as basal. Inorganic fertilizers were applied at the rate of 80: 80: $120 \mathrm{~kg} / \mathrm{ha}$ of $\mathrm{N}: \mathrm{P}_{2} \mathrm{O}_{5}: \mathrm{K}_{2} \mathrm{O}$.

Among the inorganic fertilizers, full dose of $\mathrm{P}_{2} \mathrm{O}_{5}$ and $1 / 3$ dose of $\mathrm{N}$ was applied as basal, rest $2 / 3^{\text {rd }} \mathrm{N}$ and $\mathrm{K}_{2} \mathrm{O}$ were applied in two equal splits at 45 and 90 days after planting. Observations on different morphological and yield attributing characters were recorded from five randomly selected plants from each plots. Rhizome yield per hectare was calculated on the plot weight basis. Statistical analysis of the data was done as per method suggested by Gomez and Gomez (1984). 


\section{Results and Discussion}

Perusal of the data presented in tables 1-6 revealed that there was a significant variation in the different, growth, rhizome weight and rhizome yield. Maximum plant height was recorded in the soil application of boron (as borax)@25 $\mathrm{Kg} \mathrm{ha}^{-1}(97.82 \mathrm{~cm})$ which was statistically at par with soil application of zinc (as $\left.\mathrm{ZnSO}_{4}\right) @ 25 \mathrm{Kg} \mathrm{ha}^{-1}(94.75 \mathrm{~cm})$ (Table 1).

Higher plant height was also recorded in treatment of foliar spray of zinc $\left(\right.$ as $\left.\mathrm{ZnSO}_{4}\right)$ @ $0.5 \%$ after 60 and 90 days of planting (91.37 $\mathrm{cm}$ ) and soil application of manganese (as $\left.\mathrm{MnSO}_{4}\right) @ 25 \mathrm{Kg} \mathrm{ha}^{-1}(90.25 \mathrm{~cm})$. Whereas the lowest plant height $(84.31 \mathrm{~cm})$ was recorded in control plots. With respect to tillers per plant, more number of tillers per plant was recorded with soil application of iron $\left(\right.$ as $\left.\mathrm{Fe}_{2} \mathrm{SO}_{4}\right)$ @ $25 \mathrm{Kg} \mathrm{ha}^{-1}$ (3.38) followed by soil application of iron (as $\left.\mathrm{Fe}_{2} \mathrm{SO}_{4}\right)$ @ $25 \mathrm{Kg} \mathrm{ha}^{-1}$ (3.26), foliar spray of boron (as borax) @ 0.5\% after 60 and 90 days of planting (3.19), soil application of zinc (as $\left.\mathrm{ZnSO}_{4}\right)$ @ $25 \mathrm{Kg} \mathrm{ha}{ }^{-1}$ (2.95) and soil application of boron (as borax) @ 25 $\mathrm{Kg} \mathrm{ha}^{-1}$ (2.88) and minimum number recorded in control treatment (no micronutrients) (2.16).

Soil application of manganese (as $\mathrm{MnSO}_{4}$ ) @ $25 \mathrm{Kg} \mathrm{ha}^{-1}$ showed the highest number of leaves (8.66) which was also statistically at par with soil application of zinc (as $\mathrm{ZnSO}_{4}$ ) @ $25 \mathrm{Kg} \mathrm{ha}^{-1}$ (8.41), soil application of boron (as borax) @ $25 \mathrm{Kg} \mathrm{ha}^{-1}$ (8.22) and soil application of zinc (as $\mathrm{ZnSO}_{4}$ ) @ $25 \mathrm{Kg} \mathrm{ha}^{-1}$ (8.22) and it was lowest with control (no micronutrients) treatment (7.83) (Table 2).

Maximum leaf length was recorded with soil application of boron (as borax) @ $25 \mathrm{Kg} \mathrm{ha}^{-1}$ (41.38) followed by soil application of iron (as $\mathrm{Fe}_{2} \mathrm{SO}_{4}$ ) @ $25 \mathrm{Kg} \mathrm{ha}^{-1}(41.34 \mathrm{~cm}$ ), soil application of manganese (as $\mathrm{MnSO}_{4}$ ) @ $25 \mathrm{Kg} \mathrm{ha}^{-1}(40.49 \mathrm{~cm})$, soil application of zinc (as $\mathrm{ZnSO}_{4}$ ) @ 25 $\mathrm{Kg} \mathrm{ha}^{-1} 5$ (39.96) T6 (39.95) and foliar spray of iron (as $\mathrm{Fe}_{2} \mathrm{SO}_{4}$ ) @ $0.5 \%$ after 60 and 90 days of planting $(39.74 \mathrm{~cm})$ (Table 2).

With respect to leaf breadth (Table 3), the widest leaf was recorded in the treatment of application of boron (as borax) @ $25 \mathrm{Kg} \mathrm{ha}^{-1}$ $(10.36 \mathrm{~cm})$ followed by soil application of iron $\left(\right.$ as $\left.\mathrm{Fe}_{2} \mathrm{SO}_{4}\right)$ @ $25 \mathrm{Kg} \mathrm{ha}^{-1}(10.14 \mathrm{~cm})$, foliar spray of manganese (as $\mathrm{MnSO}_{4}$ ) @ $0.5 \%$ after 60 and 90 days of planting (10.12 $\mathrm{cm})$, foliar spray of iron (as $\left.\mathrm{Fe}_{2} \mathrm{SO}_{4}\right) @ 0.5 \%$ after 60 and 90 days of planting $(9.98 \mathrm{~cm})$ and soil application of manganese (as $\mathrm{MnSO}_{4}$ ) @ $25 \mathrm{Kg} \mathrm{ha}^{-1}(9.89 \mathrm{~cm})$. The lowest leaf breadth was recorded in the treatment of soil application of zinc (as $\mathrm{ZnSO}_{4}$ ) @ 25Kg ha ${ }^{-1}$ $(8.74 \mathrm{~cm})$.

In this experiment weight of mother rhizome varied from $44.36 \mathrm{~g}$ to $66.31 \mathrm{~g}$. Maximum mother rhizome weight was recorded with soil application of iron (as $\left.\mathrm{Fe}_{2} \mathrm{SO}_{4}\right) @ 25 \mathrm{Kg}$ $\mathrm{ha}^{-1}(66.31 \mathrm{~g})$ which was also statically at par with soil application of manganese (as $\left.\mathrm{MnSO}_{4}\right) @ 25 \mathrm{Kg} \mathrm{ha}^{-1}(64.31 \mathrm{~g})$ treatment (Table 3). The lowest mother rhizome weight (44.36) was recorded in control treatment (i.e. without micronutrient). The results revealed that application micronutrient significantly increased the weight of primary and secondary rhizome per plant (Table 4).

Maximum weight of mother rhizome was record with the treatment of foliar spray of iron $\left(\right.$ as $\left.\mathrm{Fe}_{2} \mathrm{SO}_{4}\right) @ 0.5 \%$ after 60 and 90 days of planting $(115.37 \mathrm{~g})$ followed by soil application of zinc (as $\mathrm{ZnSO}_{4}$ ) @ $25 \mathrm{Kg} \mathrm{ha}^{-1}$ $(114.25 \mathrm{~g})$, soil application of manganese (as $\left.\mathrm{MnSO}_{4}\right) @ 25 \mathrm{Kg} \mathrm{ha}^{-1}(113.17 \mathrm{~g})$ and foliar spray of boron (as borax) @ $0.5 \%$ after 60 and 90 days of planting (110.63 g) (Table 4). 
Table.1 Effect of boron, manganese, zinc and iron on plant height and tiller number of turmeric

\begin{tabular}{|c|c|c|c|c|c|c|c|c|c|c|}
\hline Treatment & \multicolumn{9}{|c|}{ Plant height $(\mathrm{cm})$} & \multicolumn{5}{c|}{ Tiller Number } \\
\hline & $2010-11$ & $2011-12$ & $2012-13$ & $2015-16$ & Pooled & $2010-11$ & $2011-12$ & $2012-13$ & $2015-16$ & Pooled \\
\hline $\mathrm{T}_{1}$ & 82.00 & 80.47 & 78.83 & 95.93 & 84.31 & 2.00 & 1.88 & 1.89 & 2.87 & 2.16 \\
\hline $\mathrm{T}_{2}$ & 96.33 & 96.07 & 90.40 & 108.47 & 97.82 & 2.56 & 2.29 & 1.93 & 4.73 & 2.88 \\
\hline $\mathrm{T}_{3}$ & 94.03 & 85.23 & 78.73 & 103.00 & 90.25 & 2.56 & 1.72 & 2.11 & 3.53 & 2.48 \\
\hline $\mathrm{T}_{4}$ & 81.99 & 79.32 & 73.82 & 92.33 & 81.87 & 3.56 & 3.17 & 3.13 & 3.67 & 3.38 \\
\hline $\mathrm{T}_{5}$ & 94.40 & 91.64 & 83.81 & 109.13 & 94.75 & 3.11 & 2.50 & 2.13 & 4.07 & 2.95 \\
\hline $\mathrm{T}_{6}$ & 88.41 & 86.51 & 81.60 & 101.67 & 89.55 & 2.44 & 1.83 & 2.33 & 3.53 & 2.53 \\
\hline $\mathrm{T}_{7}$ & 86.34 & 78.35 & 73.54 & 118.00 & 89.06 & 2.56 & 1.96 & 1.97 & 3.87 & 2.59 \\
\hline $\mathrm{T}_{8}$ & 84.12 & 74.11 & 87.28 & 110.40 & 88.98 & 3.44 & 2.27 & 2.32 & 4.73 & 3.19 \\
\hline $\mathrm{T}_{9}$ & 92.57 & 84.97 & 80.13 & 107.80 & 91.37 & 3.33 & 2.69 & 2.33 & 4.67 & 3.26 \\
\hline $\mathrm{SEm} \pm$ & 3.03 & 3.66 & 3.65 & 1.74 & 1.65 & 0.21 & 0.30 & 0.31 & 0.23 & 0.13 \\
\hline $\mathrm{CD}(\mathrm{P}=0.05)$ & 9.10 & 10.97 & 10.95 & 5.20 & 4.64 & 0.64 & 0.90 & 0.92 & 0.68 & 0.37 \\
\hline $\mathrm{CV}$ & 5.99 & 7.54 & 7.82 & 2.86 & 6.35 & 12.93 & 23.04 & 23.86 & 9.93 & 15.87 \\
\hline
\end{tabular}

$\mathrm{T}_{1}$ - Control (No micronutrients), $\mathrm{T}_{2}$ - Soil application of Boron (Borax) @ $25 \mathrm{Kg} \mathrm{ha}^{-1}, \mathrm{~T}_{3}$ - Soil application of Manganese $\left(\mathrm{MnSO}_{4}\right) @ 25 \mathrm{Kg}^{-1} \mathrm{~T}_{4}-$ Soil application of Iron $\left(\mathrm{Fe}_{2} \mathrm{SO}_{4}\right) @ 25 \mathrm{Kg} \mathrm{ha}^{-1}, \mathrm{~T}_{5}$ - Soil application of Zinc $\left(\mathrm{ZnSO}_{4}\right) @ 25 \mathrm{Kg} \mathrm{ha}^{-1}, \mathrm{~T}_{6}$ - Foliar spray of Manganese $\left(\mathrm{MnSO}_{4}\right) @ 0.5 \%$ after 60 and 90 days of planting, $\mathrm{T}_{7}$ - Foliar spray of Iron $\left(\mathrm{Fe}_{2} \mathrm{SO}_{4}\right) @ 0.5 \%$ after 60 and 90 days of planting, $\mathrm{T}_{8}$ - Foliar spray of Boron (Borax) @ $0.5 \%$ after 60 and 90 days of planting and $\mathrm{T}_{9}$ - Foliar spray of Zinc $\left(\mathrm{ZnSO}_{4}\right) @ 0.5 \%$ after 60 and 90 days of planting 
Table.2 Effect of boron, manganese, zinc and iron on number of leaves and leaf length of turmeric

\begin{tabular}{|c|c|c|c|c|c|c|c|c|c|c|}
\hline \multirow[t]{2}{*}{ Treatment } & \multicolumn{5}{|c|}{ Number of leaves } & \multicolumn{5}{|c|}{ Leaf length (cm) } \\
\hline & 2010-11 & $2011-12$ & $2012-13$ & $2015-16$ & Pooled & 2010-11 & $2011-12$ & $2012-13$ & $2015-16$ & Pooled \\
\hline $\mathrm{T}_{1}$ & 7.00 & 8.50 & 8.50 & 7.07 & 7.77 & 35.10 & 40.17 & 39.60 & 35.90 & 37.69 \\
\hline $\mathrm{T}_{2}$ & 8.44 & 7.61 & 7.89 & 9.07 & 8.25 & 44.78 & 42.28 & 37.38 & 41.08 & 41.38 \\
\hline $\mathrm{T}_{3}$ & 8.33 & 9.44 & 9.00 & 7.87 & 8.66 & 43.11 & 39.78 & 39.16 & 39.92 & 40.49 \\
\hline $\mathrm{T}_{4}$ & 8.11 & 7.94 & 7.61 & 8.20 & 7.97 & 36.87 & 33.40 & 34.07 & 36.86 & 35.30 \\
\hline $\mathrm{T}_{5}$ & 8.22 & 8.50 & 8.44 & 8.47 & 8.41 & 43.89 & 40.26 & 35.59 & 40.08 & 39.96 \\
\hline $\mathrm{T}_{6}$ & 8.00 & 6.16 & 6.10 & 7.73 & 7.00 & 40.46 & 40.90 & 37.23 & 41.20 & 39.95 \\
\hline $\mathrm{T}_{7}$ & 7.89 & 7.17 & 7.33 & 9.13 & 7.88 & 40.30 & 39.70 & 36.83 & 42.12 & 39.74 \\
\hline $\mathrm{T}_{8}$ & 7.89 & 6.45 & 8.33 & 10.20 & 8.22 & 37.23 & 37.90 & 36.00 & 43.57 & 38.68 \\
\hline $\mathrm{T}_{9}$ & 8.11 & 7.11 & 6.67 & 9.33 & 7.81 & 41.72 & 40.72 & 40.23 & 42.67 & 41.34 \\
\hline $\mathrm{SEm} \pm$ & 0.36 & 0.48 & 0.49 & 0.24 & 0.21 & 1.52 & 1.36 & 1.78 & 1.07 & 0.78 \\
\hline $\mathrm{CD}(\mathrm{P}=0.05)$ & 1.09 & 1.44 & 1.46 & 0.71 & 0.60 & 4.56 & 4.09 & 5.33 & 3.21 & 2.21 \\
\hline $\mathrm{CV}$ & 7.86 & 10.87 & 10.87 & 4.76 & 9.21 & 6.52 & 5.99 & 8.24 & 4.59 & 6.88 \\
\hline
\end{tabular}

$\mathrm{T}_{1}$ - Control (No micronutrients), $\mathrm{T}_{2}$ - Soil application of Boron (Borax) @ 25Kg ha $\mathrm{K}^{-1}, \mathrm{~T}_{3}-$ Soil application of Manganese (MnSO ${ }_{4}$ @ 25Kg ha ${ }^{-1}, \mathrm{~T}_{4}-\mathrm{Soil}^{-}$ application of Iron $\left(\mathrm{Fe}_{2} \mathrm{SO}_{4}\right) @ 25 \mathrm{Kg} \mathrm{ha}^{-1}, \mathrm{~T}_{5}-$ Soil application of Zinc $\left(\mathrm{ZnSO}_{4}\right) @ 25 \mathrm{Kg} \mathrm{ha}^{-1}, \mathrm{~T}_{6}-$ Foliar spray of Manganese $\left(\mathrm{MnSO}_{4}\right) @ 0.5 \%$ after 60 and 90 days of planting, $\mathrm{T}_{7}$ - Foliar spray of Iron $\left(\mathrm{Fe}_{2} \mathrm{SO}_{4}\right) @ 0.5 \%$ after 60 and 90 days of planting, $\mathrm{T}_{8}$ - Foliar spray of Boron (Borax) @ $0.5 \%$ after 60 and 90 days of planting and $\mathrm{T}_{9}$ - Foliar spray of $\mathrm{Zinc}\left(\mathrm{ZnSO}_{4}\right) @ 0.5 \%$ after 60 and 90 days of planting. 
Table.3 Effect of boron, manganese, zinc and iron on breadth and weight of mother rhizome of turmeric

\begin{tabular}{|c|c|c|c|c|c|c|c|c|c|c|}
\hline \multirow[t]{2}{*}{ Treatment } & \multicolumn{5}{|c|}{ Leaf breadth $(\mathrm{cm})$} & \multicolumn{5}{|c|}{ Weight of mother rhizome (g) } \\
\hline & 2010-11 & 2011-12 & 2012-13 & 2015-16 & Pooled & 2010-11 & 2011-12 & $2012-13$ & 2015-16 & Pooled \\
\hline $\mathrm{T}_{1}$ & 9.00 & 9.39 & 8.39 & 8.98 & 8.94 & 40.89 & 43.22 & 42.56 & 50.77 & 44.36 \\
\hline $\mathrm{T}_{2}$ & 11.09 & 10.28 & 9.53 & 10.53 & 10.36 & 52.22 & 47.78 & 43.78 & 59.81 & 50.90 \\
\hline $\mathrm{T}_{3}$ & 10.03 & 9.83 & 9.67 & 10.02 & 9.89 & 63.00 & 62.11 & 65.11 & 67.03 & 64.31 \\
\hline $\mathrm{T}_{4}$ & 9.74 & 8.58 & 8.44 & 8.87 & 8.91 & 45.78 & 44.45 & 48.78 & 56.67 & 48.92 \\
\hline $\mathrm{T}_{5}$ & 9.52 & 8.28 & 8.11 & 9.05 & 8.74 & 48.44 & 40.44 & 43.67 & 52.56 & 46.28 \\
\hline $\mathrm{T}_{6}$ & 10.67 & 10.21 & 9.50 & 10.12 & 10.12 & 47.44 & 43.72 & 40.22 & 51.33 & 45.68 \\
\hline $\mathrm{T}_{7}$ & 10.41 & 10.08 & 8.96 & 10.47 & 9.98 & 57.56 & 53.78 & 52.17 & 60.43 & 55.98 \\
\hline $\mathrm{T}_{8}$ & 9.27 & 9.60 & 9.35 & 10.56 & 9.70 & 64.22 & 55.83 & 56.17 & 65.14 & 60.34 \\
\hline $\mathrm{T}_{9}$ & 9.61 & 10.28 & 10.22 & 10.46 & 10.14 & 64.00 & 65.94 & 66.94 & 68.37 & 66.31 \\
\hline $\mathrm{SEm} \pm$ & 0.51 & 0.74 & 0.81 & 0.18 & 0.32 & 3.29 & 2.46 & 5.0 & 1.65 & 1.64 \\
\hline $\mathrm{CD}(\mathrm{P}=0.05)$ & 1.52 & 2.22 & 2.42 & 0.55 & 0.90 & 9.87 & 7.39 & 11.98 & 4.97 & 4.63 \\
\hline $\mathrm{CV}$ & 8.82 & 13.32 & 15.32 & 3.24 & 11.50 & 10.76 & 8.41 & 16.95 & 4.06 & 1.64 \\
\hline
\end{tabular}

$\mathrm{T}_{1}$ - Control (No micronutrients), $\mathrm{T}_{2}$ - Soil application of Boron (Borax) @ 25Kg ha ${ }^{-1}, \mathrm{~T}_{3}$ - Soil application of Manganese (MnSO $)$ @ 25Kg ha ${ }^{-1}$, $\mathrm{T}_{4}-$ Soil application of Iron $\left(\mathrm{Fe}_{2} \mathrm{SO}_{4}\right) @ 25 \mathrm{Kg} \mathrm{ha}^{-1}, \mathrm{~T}_{5}$ - Soil application of Zinc $\left(\mathrm{ZnSO}_{4}\right) @ 25 \mathrm{Kg} \mathrm{ha}^{-1}, \mathrm{~T}_{6}$ - Foliar spray of Manganese $(\mathrm{MnSO} 4)$ @ $0.5 \%$ after 60 and 90 days of planting, $\mathrm{T}_{7}$ - Foliar spray of Iron $\left(\mathrm{Fe}_{2} \mathrm{SO}_{4}\right) @ 0.5 \%$ after 60 and 90 days of planting, $\mathrm{T}_{8}$ - Foliar spray of Boron (Borax) @ $0.5 \%$ after 60 and 90 days of planting and $\mathrm{T}_{9}$ - Foliar spray of Zinc $\left(\mathrm{ZnSO}_{4}\right) @ 0.5 \%$ after 60 and 90 days of planting 
Table.4 Effect of boron, manganese, zinc and iron on weight of primary and secondary rhizome of turmeric

\begin{tabular}{|c|c|c|c|c|c|c|c|c|c|c|}
\hline Treatment & \multicolumn{4}{|c|}{ Weight of primary rhizome } & \multicolumn{5}{c|}{ Weight of secondary rhizome } \\
\hline & $2010-11$ & $2011-12$ & $2012-13$ & $2015-16$ & Pooled & $2010-11$ & $2011-12$ & $2012-13$ & $2015-16$ & Pooled \\
\hline $\mathrm{T}_{1}$ & 87.00 & 71.67 & 69.00 & 90.71 & 79.59 & 80.67 & 36.22 & 35.06 & 45.95 & 49.48 \\
\hline $\mathrm{T}_{2}$ & 102.67 & 93.68 & 78.67 & 130.26 & 101.32 & 120.44 & 58.76 & 56.92 & 86.93 & 80.76 \\
\hline $\mathrm{T}_{3}$ & 112.89 & 118.50 & 107.00 & 114.29 & 113.17 & 137.56 & 56.39 & 54.39 & 79.47 & 81.95 \\
\hline $\mathrm{T}_{4}$ & 98.89 & 102.50 & 102.06 & 115.03 & 104.62 & 127.11 & 62.98 & 60.21 & 77.08 & 81.85 \\
\hline $\mathrm{T}_{5}$ & 11.78 & 114.11 & 111.94 & 119.15 & 114.25 & 110.78 & 60.67 & 58.89 & 75.45 & 76.45 \\
\hline $\mathrm{T}_{6}$ & 111.00 & 84.78 & 72.78 & 113.45 & 95.50 & 144.00 & 48.17 & 48.83 & 78.03 & 79.76 \\
\hline $\mathrm{T}_{7}$ & 153.56 & 109.61 & 103.10 & 95.21 & 115.37 & 139.00 & 58.67 & 58.61 & 55.16 & 77.86 \\
\hline $\mathrm{T}_{8}$ & 123.22 & 95.61 & 99.22 & 124.47 & 110.63 & 130.00 & 43.83 & 44.39 & 82.10 & 75.08 \\
\hline $\mathrm{T}_{9}$ & 126.33 & 81.39 & 81.94 & 119.60 & 102.32 & 144.33 & 40.94 & 42.57 & 83.04 & 77.72 \\
\hline $\mathrm{SEm} \pm$ & 4.47 & 3.55 & 5.41 & 1.97 & 1.97 & 3.74 & 2.28 & 2.86 & 1.66 & 1.39 \\
\hline $\mathrm{CD}(\mathrm{P}=0.05)$ & 13.42 & 10.65 & 16.22 & 5.91 & 5.55 & 11.21 & 6.82 & 8.58 & 5.06 & 3.91 \\
\hline $\mathrm{CV}$ & 6.82 & 6.36 & 10.21 & 3.00 & 6.56 & 5.14 & 7.60 & 9.70 & 3.96 & 6.35 \\
\hline
\end{tabular}

$\mathrm{T}_{1}$ - Control (No micronutrients), $\mathrm{T}_{2}$ - Soil application of Boron (Borax) @ 25 $\mathrm{Kg} \mathrm{ha}^{-1}, \mathrm{~T}_{3}$ - Soil application of Manganese (MnSO $\mathrm{M}_{4} @ 25 \mathrm{Kg}^{-1}, \mathrm{~T}_{4}-\mathrm{Soil}^{-1}$ application of Iron $\left(\mathrm{Fe}_{2} \mathrm{SO}_{4}\right) @ 25 \mathrm{Kg} \mathrm{ha}^{-1}, \mathrm{~T}_{5}$ - Soil application of Zinc $\left(\mathrm{ZnSO}_{4}\right) @ 25 \mathrm{Kg} \mathrm{ha}^{-1}, \mathrm{~T}_{6}$ - Foliar spray of Manganese $\left(\mathrm{MnSO}_{4}\right) @ 0.5 \%$ after 60 and 90 days of planting, $\mathrm{T}_{7}$ - Foliar spray of Iron $\left(\mathrm{Fe}_{2} \mathrm{SO}_{4}\right) @ 0.5 \%$ after 60 and 90 days of planting, $\mathrm{T}_{8}$ - Foliar spray of Boron (Borax) @ $0.5 \%$ after 60 and 90 days of planting and $\mathrm{T}_{9}$ - Foliar spray of Zinc $\left(\mathrm{ZnSO}_{4}\right) @ 0.5 \%$ after 60 and 90 days of planting 


\section{Int.J.Curr.Microbiol.App.Sci (2017) 6(5): 1471-1482}

Table.5 Effect of boron, manganese, zinc and iron on plant population of turmeric

\begin{tabular}{|c|c|c|c|c|c|c|c|c|c|c|}
\hline \multirow[t]{2}{*}{ Treatment } & \multicolumn{5}{|c|}{ Plant population at (30DAP) } & \multicolumn{5}{|c|}{ Plant population at (60DAP) } \\
\hline & 2010-11 & 2011-12 & $2012-13$ & $2015-16$ & Pooled & 2010-11 & 2011-12 & $2012-13$ & $2015-16$ & Pooled \\
\hline $\mathrm{T}_{1}$ & 36.00 & 37.67 & 37.67 & 39.33 & 37.67 & 35.00 & 35.67 & 35.67 & 38.67 & 36.25 \\
\hline $\mathrm{T}_{2}$ & 38.67 & 34.67 & 35.00 & 38.67 & 36.75 & 38.67 & 34.00 & 34.00 & 36.67 & 35.83 \\
\hline $\mathrm{T}_{3}$ & 39.33 & 34.67 & 36.67 & 37.67 & 37.08 & 38.33 & 34.33 & 36.33 & 36.67 & 36.42 \\
\hline $\mathrm{T}_{4}$ & 38.33 & 36.33 & 36.67 & 38.00 & 37.33 & 36.33 & 35.67 & 36.33 & 36.67 & 36.25 \\
\hline $\mathrm{T}_{5}$ & 37.33 & 36.67 & 37.00 & 38.33 & 37.33 & 36.67 & 35.33 & 37.00 & 38.00 & 36.75 \\
\hline $\mathrm{T}_{6}$ & 38.67 & 38.00 & 38.33 & 37.67 & 38.17 & 36.67 & 37.33 & 38.00 & 37.00 & 37.25 \\
\hline $\mathrm{T}_{7}$ & 39.67 & 35.67 & 36.00 & 37.67 & 37.25 & 36.67 & 35.33 & 35.33 & 37.67 & 36.25 \\
\hline $\mathrm{T}_{8}$ & 40.00 & 39.67 & 39.00 & 38.00 & 39.17 & 37.33 & 38.00 & 38.33 & 38.00 & 37.92 \\
\hline $\mathrm{T}_{9}$ & 37.33 & 38.67 & 38.33 & 39.67 & 38.50 & 37.33 & 37.33 & 38.00 & 39.67 & 38.08 \\
\hline SEm \pm & 0.81 & 1.13 & 1.00 & 0.86 & 0.51 & 1.09 & 1.14 & 0.93 & .71 & 0.51 \\
\hline $\mathrm{CD}(\mathrm{P}=0.05)$ & 2.45 & 3.38 & 3.01 & 2.59 & 1.44 & 3.28 & 3.43 & 2.80 & 2.14 & 1.43 \\
\hline $\mathrm{CV}$ & 3.69 & 5.30 & 4.68 & 3.91 & 4.68 & 5.12 & 5.51 & 4.42 & 3.28 & 4.77 \\
\hline
\end{tabular}

$\mathrm{T}_{1}$ - Control (No micronutrients), $\mathrm{T}_{2}$ - Soil application of Boron (Borax) @ 25Kg ha $\mathrm{K}^{-1}, \mathrm{~T}_{3}-$ Soil application of Manganese (MnSO ${ }_{4}$ @ 25Kg ha ${ }^{-1}, \mathrm{~T}_{4}-\mathrm{Soil}^{-}$ application of Iron $\left(\mathrm{Fe}_{2} \mathrm{SO}_{4}\right) @ 25 \mathrm{Kg} \mathrm{ha}^{-1}, \mathrm{~T}_{5}-$ Soil application of Zinc $\left(\mathrm{ZnSO}_{4}\right) @ 25 \mathrm{Kg} \mathrm{ha}^{-1}, \mathrm{~T}_{6}-$ Foliar spray of Manganese $\left(\mathrm{MnSO}_{4}\right) @ 0.5 \%$ after 60 and 90 days of planting, $\mathrm{T}_{7}$ - Foliar spray of Iron $\left(\mathrm{Fe}_{2} \mathrm{SO}_{4}\right) @ 0.5 \%$ after 60 and 90 days of planting, $\mathrm{T}_{8}$ - Foliar spray of Boron (Borax) @ $0.5 \%$ after 60 and 90 days of planting and $\mathrm{T}_{9}$ - Foliar spray of $\mathrm{Zinc}\left(\mathrm{ZnSO}_{4}\right) @ 0.5 \%$ after 60 and 90 days of planting. 
Table.6 Effect of boron, manganese, zinc and iron on rhizome yield of turmeric

\begin{tabular}{|c|c|c|c|c|c|c|c|c|c|c|}
\hline Treatment & \multicolumn{9}{|c|}{ Yield per Plot $\left(\mathbf{k g} / \mathbf{3 ~ m}^{\mathbf{2}}\right)$} & \multicolumn{5}{c|}{ Projected Yield (t/ha) } \\
\hline & $2010-11$ & $2011-12$ & $2012-13$ & $2015-16$ & Pooled & $2010-11$ & $2011-12$ & $2012-13$ & $2015-16$ & Pooled \\
\hline $\mathrm{T}_{1}$ & 6.89 & 6.70 & 6.50 & 8.57 & 7.17 & 13.89 & 13.51 & 13.10 & 17.28 & 14.45 \\
\hline $\mathrm{T}_{2}$ & 10.17 & 10.33 & 10.17 & 13.87 & 11.13 & 20.50 & 20.83 & 20.50 & 27.96 & 22.45 \\
\hline $\mathrm{T}_{3}$ & 9.33 & 7.60 & 7.93 & 11.78 & 9.16 & 18.82 & 15.32 & 15.99 & 23.74 & 18.47 \\
\hline $\mathrm{T}_{4}$ & 8.67 & 7.83 & 7.90 & 11.61 & 9.00 & 17.47 & 15.79 & 15.93 & 23.41 & 18.15 \\
\hline $\mathrm{T}_{5}$ & 9.33 & 7.73 & 8.00 & 11.40 & 9.11 & 18.82 & 15.59 & 16.13 & 22.98 & 18.38 \\
\hline $\mathrm{T}_{6}$ & 8.83 & 8.37 & 7.57 & 11.15 & 8.98 & 17.81 & 16.87 & 15.25 & 22.47 & 18.10 \\
\hline $\mathrm{T}_{7}$ & 7.50 & 6.37 & 5.83 & 9.80 & 7.38 & 15.12 & 12.84 & 11.76 & 19.75 & 14.87 \\
\hline $\mathrm{T}_{8}$ & 8.83 & 10.03 & 10.17 & 13.35 & 10.59 & 17.81 & 20.23 & 20.50 & 26.91 & 21.36 \\
\hline $\mathrm{T}_{9}$ & 9.83 & 7.97 & 8.47 & 13.21 & 9.87 & 19.82 & 16.06 & 17.07 & 26.64 & 19.90 \\
\hline $\mathrm{SEm} \pm$ & 0.71 & 0.73 & 0.79 & 0.25 & 0.43 & 1.42 & 1.48 & 1.60 & 0.50 & 0.86 \\
\hline $\mathrm{CD}(\mathrm{P}=0.05)$ & 2.11 & 2.20 & 2.38 & 0.76 & 1.20 & 4.26 & 4.43 & 4.80 & 1.52 & 2.42 \\
\hline $\mathrm{CV} \%$ & 13.84 & 15.68 & 17.07 & 3.75 & 16.10 & 13.84 & 15.68 & 17.64 & 3.75 & 16.09 \\
\hline
\end{tabular}

$\mathrm{T}_{1}$ - Control (No micronutrients), $\mathrm{T}_{2}$ - Soil application of Boron (Borax)@ $25 \mathrm{Kg} \mathrm{ha}^{-1}, \mathrm{~T}_{3}$ - Soil application of Manganese $\left(\mathrm{MnSO}_{4}\right) @ 25 \mathrm{Kg} \mathrm{ha}^{-1}, \mathrm{~T}_{4}-\mathrm{Soil}$ application of Iron $\left(\mathrm{Fe}_{2} \mathrm{SO}_{4}\right) @ 25 \mathrm{Kg} \mathrm{ha}^{-1}, \mathrm{~T}_{5}$ - Soil application of Zinc $\left(\mathrm{ZnSO}_{4}\right) @ 25 \mathrm{Kg} \mathrm{ha}^{-1}, \mathrm{~T}_{6}-$ Foliar spray of Manganese $\left(\mathrm{MnSO}_{4}\right) @ 0.5 \%$ after 60 and 90 days of planting, $\mathrm{T}_{7}$ - Foliar spray of Iron $\left(\mathrm{Fe}_{2} \mathrm{SO}_{4}\right) @ 0.5 \%$ after 60 and 90 days of planting, $\mathrm{T}_{8}$ - Foliar spray of Boron (Borax) @ $0.5 \%$ after 60 and 90 days of planting and $\mathrm{T}_{9}$ - Foliar spray of Zinc $\left(\mathrm{ZnSO}_{4}\right) @ 0.5 \%$ after 60 and 90 days of planting. 
Table.7 Cost Benefit ratio of different treatments

\begin{tabular}{|c|c|c|c|c|c|c|c|c|c|c|c|c|c|c|}
\hline \multirow{3}{*}{$\begin{array}{l}\text { Treat } \\
\text { ments }\end{array}$} & \multicolumn{9}{|c|}{ Expense (Rs./ha) } & \multicolumn{3}{|c|}{ Income (Rs./ha) } & \multirow{3}{*}{$\begin{array}{c}\text { Net } \\
\text { income } \\
\text { (Rs./ha) }\end{array}$} & \multirow[t]{3}{*}{ C:B } \\
\hline & \multirow{2}{*}{$\begin{array}{c}\text { Land } \\
\text { Preparation }\end{array}$} & \multirow{2}{*}{$\begin{array}{c}\text { Seed } \\
\text { rhizome } \\
\text { cost }\end{array}$} & \multirow{2}{*}{$\begin{array}{l}\text { Micronu } \\
\text { trient } \\
\text { and } \\
\text { applicati } \\
\text { on cost }\end{array}$} & \multirow{2}{*}{$\begin{array}{l}\text { Labour (Bed } \\
\text { preparation, } \\
\text { sowing, weeding, } \\
\text { harvesting, } \\
\text { spraying etc) }\end{array}$} & \multicolumn{4}{|c|}{ Fertilizer } & \multirow[t]{2}{*}{ Total } & \multirow{2}{*}{$\begin{array}{l}\text { Producti } \\
\text { on (ton/ } \\
\text { ha) }\end{array}$} & \multirow{2}{*}{$\begin{array}{c}\text { Rate } \\
\text { (Rs./ } \\
\text { t) }\end{array}$} & \multirow{2}{*}{$\begin{array}{c}\text { Sell } \\
\text { amount } \\
\text { (Rs.) }\end{array}$} & & \\
\hline & & & & & $\mathbf{N}$ & $\mathbf{P}$ & $\mathbf{K}$ & FYM & & & & & & \\
\hline $\mathrm{T} 1$ & 10000 & 50,000 & 0 & $1,25,572$ & 1,056 & 3,075 & 3,206 & 22,500 & $2,15,409$ & 14.45 & 20,000 & 289000 & 73,591 & $1: 1.34$ \\
\hline $\mathrm{T} 2$ & 10000 & 50,000 & 5,512 & $1,25,572$ & 1,056 & 3,075 & 3,206 & 22,500 & $2,20,921$ & 22.45 & 20,000 & 449000 & $2,28,079$ & $1: 2.03$ \\
\hline T3 & 10000 & 50,000 & 2,512 & $1,25,572$ & 1,056 & 3,075 & 3,206 & 22,500 & $2,17,921$ & 18.47 & 20,000 & 369400 & $1,51,479$ & $1: 1.70$ \\
\hline $\mathrm{T} 4$ & 10000 & 50,000 & 1,137 & $1,25,572$ & 1,056 & 3,075 & 3,206 & 22,500 & $2,16,546$ & 18.15 & 20,000 & 363000 & $1,46,454$ & $1: 1.68$ \\
\hline T6 & 10000 & 50,000 & 2,528 & $1,25,572$ & 1,056 & 3,075 & 3,206 & 22,500 & $2,17,937$ & 18.1 & 20,000 & 362000 & $1,44,063$ & $1: .66$ \\
\hline $\mathrm{T} 7$ & 10000 & 50,000 & 2,198 & $1,25,572$ & 1,056 & 3,075 & 3,206 & 22,500 & $2,17,607$ & 14.87 & 20,000 & 297400 & 79,793 & $1: 1.37$ \\
\hline T8 & 10000 & 50,000 & 3,284 & $1,25,572$ & 1,056 & 3,075 & 3,206 & 22,500 & $2,18,693$ & 21.36 & 20,000 & 427200 & $2,08,507$ & $1: 1.95$ \\
\hline $\mathrm{T}_{9}$ & 10000 & 50,000 & 2,384 & $1,25,572$ & 1,056 & 3,075 & 3,206 & 22,500 & $2,17,793$ & 19.9 & 20,000 & 398000 & $1,80,207$ & $1: 1.83$ \\
\hline
\end{tabular}

$\mathrm{T}_{1}$ - Control (No micronutrients), $\mathrm{T}_{2}$ - Soil application of Boron (Borax) @ 25Kg ha ${ }^{-1}, \mathrm{~T}_{3}$ - Soil application of Manganese $\left(\mathrm{MnSO}_{4}\right) @ 25 \mathrm{Kg} \mathrm{ha}^{-1}, \mathrm{~T}_{4}-\mathrm{Soil}^{-1}$ application of Iron $\left(\mathrm{Fe}_{2} \mathrm{SO}_{4}\right) @ 25 \mathrm{Kg} \mathrm{ha}^{-1}, \mathrm{~T}_{5}$ - Soil application of Zinc $\left(\mathrm{ZnSO}_{4}\right) @ 25 \mathrm{Kg} \mathrm{ha}^{-1}, \mathrm{~T}_{6}$ - Foliar spray of Manganese $\left(\mathrm{MnSO}_{4}\right) @ 0.5 \%$ after 60 and 90 days of planting, $\mathrm{T}_{7}$ - Foliar spray of Iron $\left(\mathrm{Fe}_{2} \mathrm{SO}_{4}\right) @ 0.5 \%$ after 60 and 90 days of planting, $\mathrm{T}_{8}$ - Foliar spray of Boron (Borax) @ $0.5 \%$ after 60 and 90 days of planting and $\mathrm{T}_{9}$ - Foliar spray of Zinc $\left(\mathrm{ZnSO}_{4}\right) @ 0.5 \%$ after 60 and 90 days of planting 
With respect to the weight of secondary rhizome, the higher weight of secondary rhizome was recorded with the treatment of soil application of manganese (as $\left.\mathrm{MnSO}_{4}\right)$ @ $25 \mathrm{Kg} \mathrm{ha}^{-1}(81.95 \mathrm{~g})$, - soil application of iron (as $\left.\mathrm{Fe}_{2} \mathrm{SO}_{4}\right) @ 25 \mathrm{Kg} \mathrm{ha}^{-1}(81.85 \mathrm{~g}$ ) and soil application of boron (as borax) @ $25 \mathrm{Kg} \mathrm{ha}^{-1}$ (80.76 g). Lowest primary and secondary rhizome weight was recorded in the control treatment (79.59 $\mathrm{g}$ and $49.48 \mathrm{~g}$, respectively) (Table 4). Effect of micronutrient on the turmeric plant population survival from 30 days after transplanting to the maturity (i.e. at the time harvest) was almost nothing (Table $5)$.

Among the different treatment, soil application of boron (as borax) @ $25 \mathrm{Kg} \mathrm{ha}^{-1}$ gave the highest yield $\left(11.13 \mathrm{~kg} / 3 \mathrm{~m}^{2}\right.$ and $22.45 \mathrm{t} / \mathrm{ha}$ ) which was also statistically at par with foliar spray of boron (as borax) @ $0.5 \%$ after 60 and 90 days of planting treatment (10.59 kg/3m $\mathrm{m}^{2}$ and $21.36 \mathrm{t} / \mathrm{ha}$ ) (Table 4). Higher yield of turmeric rhizome was also recorded in the treatment of soil application of iron (as $\mathrm{Fe}_{2} \mathrm{SO}_{4}$ ) @ $25 \mathrm{Kg} \mathrm{ha}^{-1}(9.87$ $\mathrm{kg} / 3 \mathrm{~m} 2$ and $19.90 \mathrm{t} / \mathrm{ha})$. The lowest yield was recorded in the control plot $(7.17 \mathrm{~kg} / 3 \mathrm{~m} 2$ and $14.45 \mathrm{t} / \mathrm{ha})$. In this experiment higher yield recorded in micronutrient treated plot as compared to the control plots. This might be due the beneficial effect of the micronutrient on turmeric yield. Among the different micronutrient, soil application of boron gave highest yield as compared to the other treatment. Sugtto and Mafutuchah (1995), Pawar and Gavande (1992) and Singh et al., (1992) also observed similar findings in their report as found in boron application.

Considering cost benefit ratio, the highest $\mathrm{C}$ : $\mathrm{B}$ ratio $(1: 2.04)$ was recorded with the soil application of boron (as borax) @ $25 \mathrm{Kg} \mathrm{ha}^{-1}$ followed by foliar spray of boron $(1: 1.95)$ (as borax) @ $0.5 \%$ at 60 and 90 days after planting and lowest $\mathrm{C}: \mathrm{B}$ ratio $(1: 1.34)$ was recorded in the control treatment (i.e. application of no micronutrient) (Table 7).

In conclusion, from the above discussion it may be concluded that soil application of boron (as borax) @ 25Kg ha ${ }^{-1}$ ) and foliar spray of boron (as borax) @ $0.5 \%$ at 60 and 90 days after planting (1: 1.95) were beneficial for getting higher yield in turmeric.

\section{Acknowledgement}

The authors are highly indebted to Indian Institute of Spices Research-Indian Council of Agricultural Research, for their financial help by providing research grant, guidance and support in every step of experiment.

\section{References}

Anonymous. 2012. Major State wise Area \& production of Spices in India, accessed at

<http://www.indianspices.com/statistics $>$ on 20.06.2013.

Banafar, R.N.S. and Tiwari, RJ. 1995. Response of turmeric to potassium application in medium black soils of Madhya pradesh. Crop Res., (Hissar), 10: 93-95.

Bose, P., Sanyal, D. and Majumdar, K. 2008. Balancing sulfur and magnesium nutrition for turmeric and carrot grown on red lateritic soil. Better Crops, 92(1): 23-25.

Chandra, R., Desai, A.R. and Govind, S. 1997. Effect of foliar spray of magnesium and planting materials on growth and yield of turmeric. J. Hill Res., 10(1): 1-4.

Chhibba, I.M., Nayyar, V.K., Kanwar, J.S. 2007. Influence of mode and source of applied iron on fenugreek (Trigonella corniculata L). in a Typic Ustochrept in Punjab, India. Int. J. Agri. Biol., 9(2): 254-256. 
Devi, K.S.P. and Sangamithra, A. 2011. Turmeric - Indian Saffron, Technical Bulletin: Science Tech Entrepreneur, Pp. 1-7.

Gomez, K.A. and Gomez, A.A. 1984. Statistical Procedures for Agricultural Research $\left(2^{\text {nd }}\right.$ Ed.. A Willey-Inter Science Publication (John Wiley and sons) New York, Pp. 20-30.

Gupta, C.R. And Singar, S.S. 1998. Effect of varying levels of of nitrogen, phosphorus and potassium level on growth and yield of turmeric in hill zone,Karnataka. J. Spices and Aromatic Crops, $9: 28-32$

Halder, N.K., Shill, N.C., Siddiky, M.A., Sarkar, J. and Gomes, R. 2007. Response of turmeric to zinc and boron fertilization. J. Biol. Sci., 7(1): 182-187.

Kavitha, P.R. 2012. Nutrient management for yield and quality improvement in kacholam (Kaempferia galanga L). M.Sc. (Ag). thesis, Kerala Agricultural University, Thrissur, 97.

Pawar, H.K. and Gavande, S.S. 1992. Content and uptake of NPK by ginger rhizomes as influenced by irrigation and nitrogen management. J. Maharastra Agri. Univ., 17: 282-283.

Roy, A., Cahatterjee, R., Hassan, A. and Maitra, S.K. 1992. Effect of Zn, Fe and $\mathrm{B}$ on growth, yield and nutrient content in leaf of ginger. Indian Cocoa, Arecanut and Spices J., 15: 99-101.

Singh, V.P., Swer, B. and Singh, S.P. 1992. Influence of nitrogen and potassium on yield of turmeric. Indian Cocoa, Arecanut and Spices J., 15: 104-106.

Sugtto, Y. And Mafutuchah. 1995. Influence of rates of farmyard manure and $\mathrm{KCl}$ on growth, yield and quality of ginger rhizome. Agrivita, 18: 67-73.

\section{How to cite this article:}

Datta, S., S. Chakraborty, J.C. Jana, A. Debnath, M.K. Roy and Haque, S. 2017. Effect of Different Micronutrients on Turmeric Variety Suranjana in Terai Region of West Bengal, India. Int.J.Curr.Microbiol.App.Sci. 6(5): 1471-1482. doi: https://doi.org/10.20546/ijcmas.2017.605.160 